Article

\title{
In Silico Analysis of Gene Expression Change Associated with Copy Number of Enhancers in Pancreatic Adenocarcinoma
}

\author{
Rajesh Kumar ${ }^{1,2}$, Sumeet Patiyal ${ }^{1}$, Vinod Kumar ${ }^{1,2}$, Gandharva Nagpal ${ }^{2}$ \\ and Gajendra P.S. Raghava $1,2, *$ \\ 1 Department of Computational Biology, Indraprastha Institute of Information Technology, Delhi 110020, India \\ 2 Bioinformatics Centre, CSIR-Institute of Microbial Technology, Chandigarh 160036, India \\ * Correspondence: raghava@iiitd.ac.in; Tel.: +91-11-269-0744
}

Received: 5 May 2019; Accepted: 11 July 2019; Published: 22 July 2019

check for updates

\begin{abstract}
Understanding the gene regulatory network governing cancer initiation and progression is necessary, although it remains largely unexplored. Enhancer elements represent the center of this regulatory circuit. The study aims to identify the gene expression change driven by copy number variation in enhancer elements of pancreatic adenocarcinoma (PAAD). The pancreatic tissue specific enhancer and target gene data were taken from EnhancerAtlas. The gene expression and copy number data were taken from The Cancer Genome Atlas (TCGA). Differentially expressed genes (DEGs) and copy number variations (CNVs) were identified between matched tumor-normal samples of PAAD. Significant CNVs were matched onto enhancer coordinates by using genomic intersection functionality from BEDTools. By combining the gene expression and CNV data, we identified 169 genes whose expression shows a positive correlation with the CNV of enhancers. We further identified 16 genes which are regulated by a super enhancer and 15 genes which have high prognostic potential ( $Z$-score $>1.96$ ). Cox proportional hazard analysis of these genes indicates that these are better predictors of survival. Taken together, our integrative analytical approach identifies enhancer CNV-driven gene expression change in PAAD, which could lead to better understanding of PAAD pathogenesis and to the design of enhancer-based cancer treatment strategies.
\end{abstract}

Keywords: enhancer; copy number variation; regulatory elements; pancreatic cancer; adenocarcinoma; super enhancer; survival; differential expressed genes; functional enrichment analysis

\section{Introduction}

Cancer is a genetic disease, as initiation, progression, and metastasis are governed by several genetic and epigenetic changes within the genome. Pancreatic cancer is a leading cause of mortality in the Western world [1]. Patients are mostly diagnosed at an advanced stage, resulting in poor response to therapy. The 5-year survival rate of pancreatic cancer patients is $6 \%$. This is the worst survival rate among all 22 common types of cancer [2]. Cancer cells harbor thousands of genomic alterations, including amplification, deletion, insertion, translocation, transversion and copy number variation $(\mathrm{CNV})$. Only a small fraction of these genomic alterations represents the driver mutations that are truly morbific. Often biological function of a cell is maintained by a complex multi-level gene regulatory hierarchy, including post-translation modification, enhancer activation, miRNA mediated gene regulation, and RNA editing. In cancer cells, these regulatory circuits are disrupted or rewired, which leads to the disease phenotype [3].

Whole genome sequencing of tumor and normal samples provides unprecedented data that can be used to identify mutations associated with disease. The Cancer Genome Atlas (TCGA) [4] and 
International Cancer Genome Consortium (ICGC) are such large-scale efforts [5]. These projects and follow up studies identified several mutated genes and pathways, and have significantly increased our knowledge of cancer, leading to the discovery of new targets, diagnoses, prognoses and improvements of therapy. Mutation in the coding region of several genes has been consistently identified by several studies, such as with altered frequency (e.g., KRAS, TP53) or loss (e.g., CDNK2A, SMAD4, ROBO2), and altered pathways, such as Wnt/Notch. Thus far, cancer studies have mostly focused on characterizing the functional impact of mutations in protein-coding sequences. COSMIC (Catalogue of Somatic Mutation in Cancer) is one such effort, which lists only aberrations in the coding sequence of the genes [6].

The coding fraction represents only $2 \%$ of the genome. The remaining $98 \%$ the of human genome is transcribed either in non-coding RNA or into regulatory elements. Most of the genomic alteration resides in the non-coding region of the genome [7]. It is also observed that more than $80 \%$ of genetic variants associated with diseases were observed in the non-coding region of the genome [8]. Nevertheless, this portion of the genome has been largely unexplored. Mutation in the non-coding region of the genome can modify the function of both cis- and trans-acting elements in a regulatory circuit $[9,10]$. Therefore, this can lead to the development of cell behavior towards tumorigenesis [11]. However, our knowledge of gene regulatory circuit rewiring is far from complete. Existing research shows the importance of the enhancers as a key piece in this gene regulation circuit [12].

Enhancers are DNA elements of up to 50-1500 base pairs (bp) [13]. They interact with their target promoters irrespective of their position to regulate downstream gene expression [14]. Several studies link alteration within the enhancer to disease phenotype, e.g., DiseaseEnhancer is one such database containing information on 847 disease associated enhancers in 143 human diseases [15]. Several studies confirm the dosage effect of copy number variation in gene expression [16,17]. The dosage effect of loss/gain in enhancer elements is further supported by experimental evidence. Zhang X., et al. [18] demonstrate that duplication in the enhancer region results in higher gene expression by luciferase assay. Experimental studies also confirm that copy number alterations within the enhancer element are an important driver of tumorigenesis. In head and neck squamous cell carcinoma, KLF5 was upregulated by amplification super-enhancers marked by H3K27ac [19].

In this study, we represent an integrative approach that embellishes the relationship between enhancer $\mathrm{CNV}$ and targeting gene expression changes in pancreatic adenocarcinoma. The following are main goals of this study: (i) identification of enhancers having a significant change in their CNVs; (ii) understanding the effect of a change in enhancer $\mathrm{CNVs}$ on the expression of associated genes; (iii) understanding the clinical potential of genes regulated by CNVs of enhancers; and, (iv) pathway enrichment analysis of enhancer associated genes. In order to achieve our objectives, we performed the following steps. Firstly, coordinates of enhancers specific to pancreatic tissue, as well as the associated genes of these enhancers, were obtained from EnhancerAtlas [20]. EnhancerAtlas provides high quality experimental data on enhancers and cross validation for each cell/tissue type was done by integration of the multiple experimental dataset [21]. Secondly, gene expression and CNV data of 185 patients were obtained from TCGA. Thirdly, the software GISTIC was used for identifying CNVs having a significant change (loss/gain) in PAAD samples. Fourthly, we used BEDTools to identify enhancers specific to pancreatic tissue having significant variation in their CNVs in PAAD samples. These enhancers were further classified based on their increase or decrease in CNVs and based on positive or negative correlation with associated genes. Finally, we performed a wide range of analyses to understand the effect of enhancers on gene regulation, with the potential to classify high and low risk patients, and the rewiring of regulation in cancer. 


\section{Results}

\subsection{Identification of Enhancers Having Change in CNV}

In total, 952 significant CNV regions were obtained for the pancreatic cancer sample, out of which 321 regions were amplified and 631 regions were deleted (Table S1). Based on the genomic coordinate, by employing genomic intersecting functionality from BEDTools, we mapped enhancer coordinates on to the significant CNV region. Finally, 421 enhancers having a significant variation in their CNV region (amplified/increase or deleted/decreased) were mapped on to enhancer coordinates (Table S2). The gene expression analysis of quantile normalized pancreatic cancer vs normal samples identified 2431 upregulated and 3614 downregulated genes. By checking the expression of enhancer target genes in the same TCGA sample, we identified 169 concordant regulatory pairs, which reflected a CNV-based enhancer dosage effect on gene expression (Table S3). This regulatory pair consisted of 89 upregulated and 80 downregulated genes; all 169 gene expressions showed a positive correlation with the CNV of enhancers. Our analysis in this study mainly focused on these 169 genes, and considered two possible concordant changes: enhancer copy number gain/gene upregulation and copy number loss/gene downregulation.

\subsection{Pathway Enrichment Analysis of Genes Regulated by Enhancers}

In order to gain further insight into the enhancer CNV-driven differentially expressed genes, gene interaction network and enrichment analyses were performed using STRING (Search Tool for the Retrieval of Interacting Genes/Proteins) and Enrichr, respectively. As shown in Figure 1, upregulated genes RPS16, SCAMP3 and MUC1 show three or more interaction direct interactions. These genes are the major components regulating the glucose metabolism and proteolysis in pancreatic cells [22,23], indicative of poor prognosis [24] and involved in PDGFA expression in pancreatic cancer progression, respectively [25]. Figure 2 (for downregulated) shows UBB, PRPF8, ERLIN and RPS6 genes involved in direct interaction. The $U B B$ gene is involved in TNF- $\alpha$ induced NF- $\kappa B$ activation, and thus plays a role in the stabilization of the tumor suppressor P53 gene. Thus, downregulation of the UBB gene by any means may serve as an anti-tumor treatment [26]. RPS6 and ERLIN1 genes are involved in the mTOR signaling pathway, which is essential for cell growth and metabolism, particularly tumor formation and angiogenesis $[27,28]$. Pathway enrichment analysis of the concordant gene set provides clues regarding their role in regulating cell cycle progression, and the Eph cell signaling pathway (Figure 3). This pathway regulates kinase activity, which is frequently mutated in almost all types of cancer [29]. GO (Gene Ontology) enrichment analysis was performed to identify the most correlative biological, molecular and cellular function of the genes. GO analysis demonstrates that the genes are enhanced in receptor binding and in regulation of the apoptotic pathway. The detailed results are presented in Figure 4.

\subsection{Cox Proportional Hazard Analysis of Enhancer Regulated Genes}

Univariate analysis of genes was done using a Cox proportional hazard regression model on the mean and median cut off of gene expression across the patient samples. On performing the analysis, 41 out of 169 genes were found to be significant on the basis of $p$-value $<0.05$, which is statistically significant. Twenty-nine genes had a hazard ratio of more than 1.5, that is, the expression of these genes results in the progression of cancer at the rate of more than 1.5 in patients belonging to a high-risk group [expression value $<$ median(gene expression)] as compared to the low-risk group [expression value $>$ median(gene expression)]. Table 1 Shows the top 15 genes. VAMP2 has a HR of 2.9, and downregulation of genes is involved in acute acinar pancreatitis [30]. The CERK gene, with a HR of 2.48 , is involved in metastasizing of pancreatic cancer cells and thus leads to cancer development at distant places [31]. 


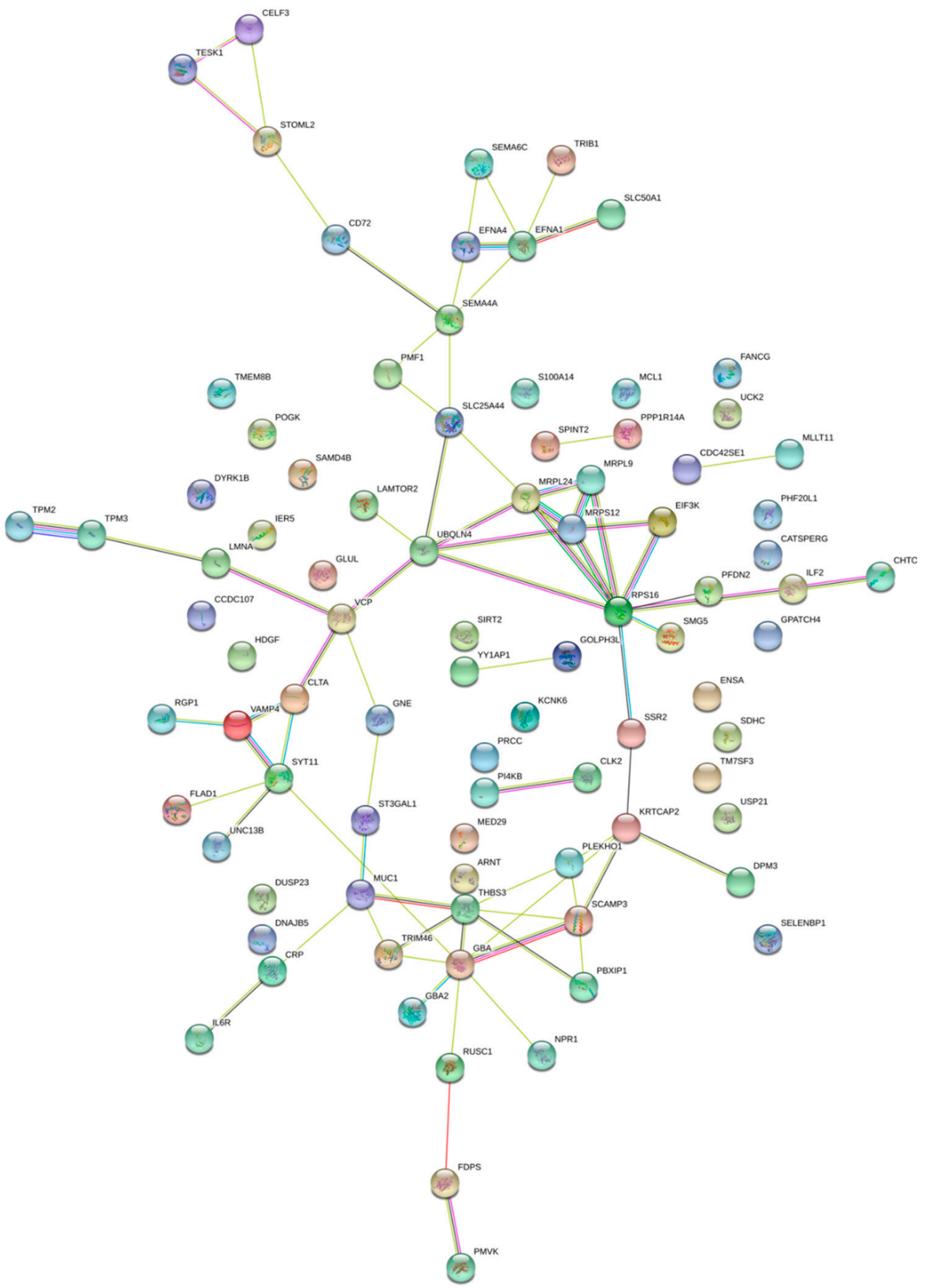

Figure 1. Gene interaction network of genes upregulated due to copy number gain in their regulatory enhancer region. Nodes represent proteins and edges represent protein-protein associations. 


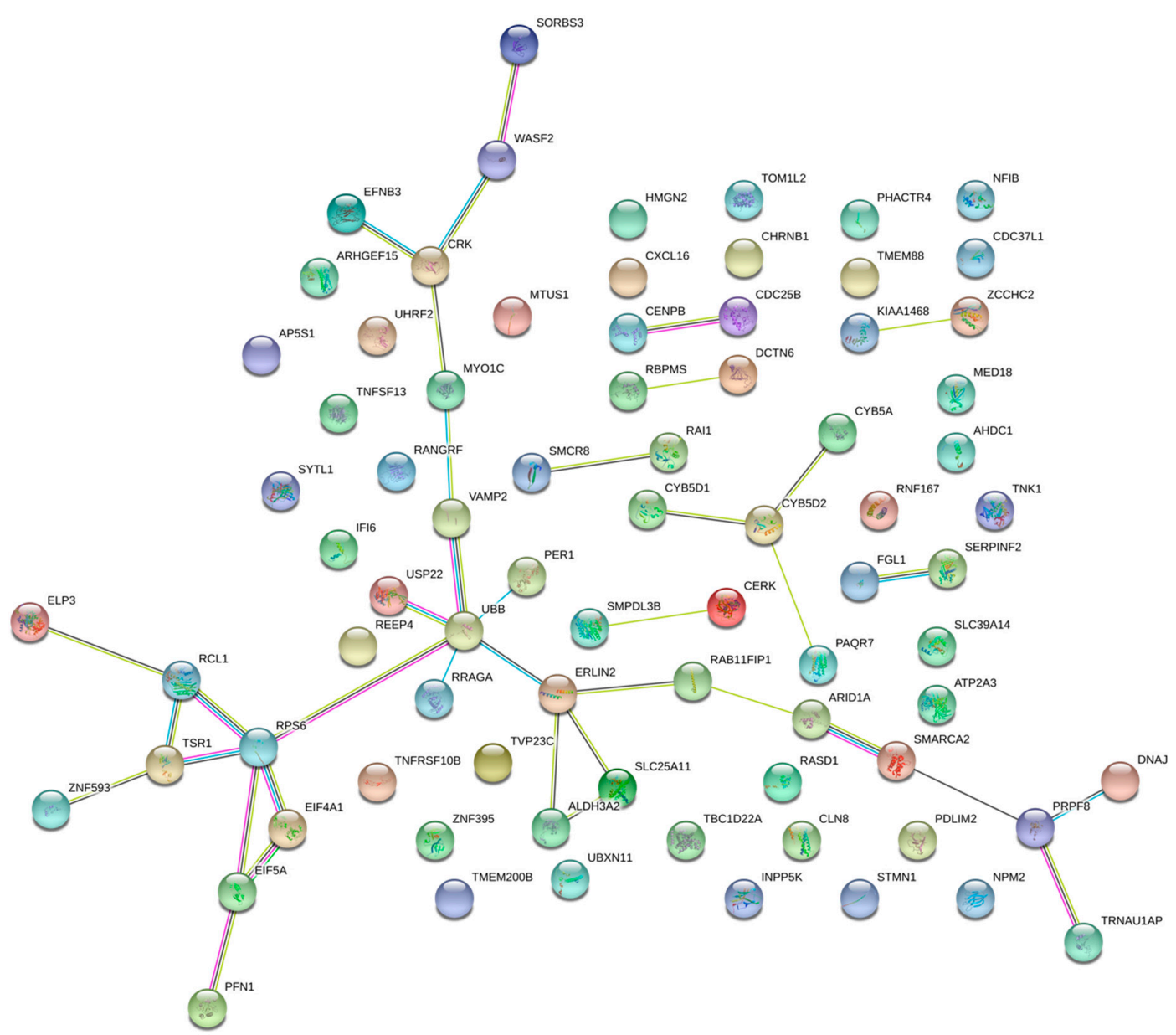

Figure 2. Gene interaction network of genes downregulated due to copy number loss in their regulatory enhancer region. Nodes represent proteins and edges represent protein-protein associations.

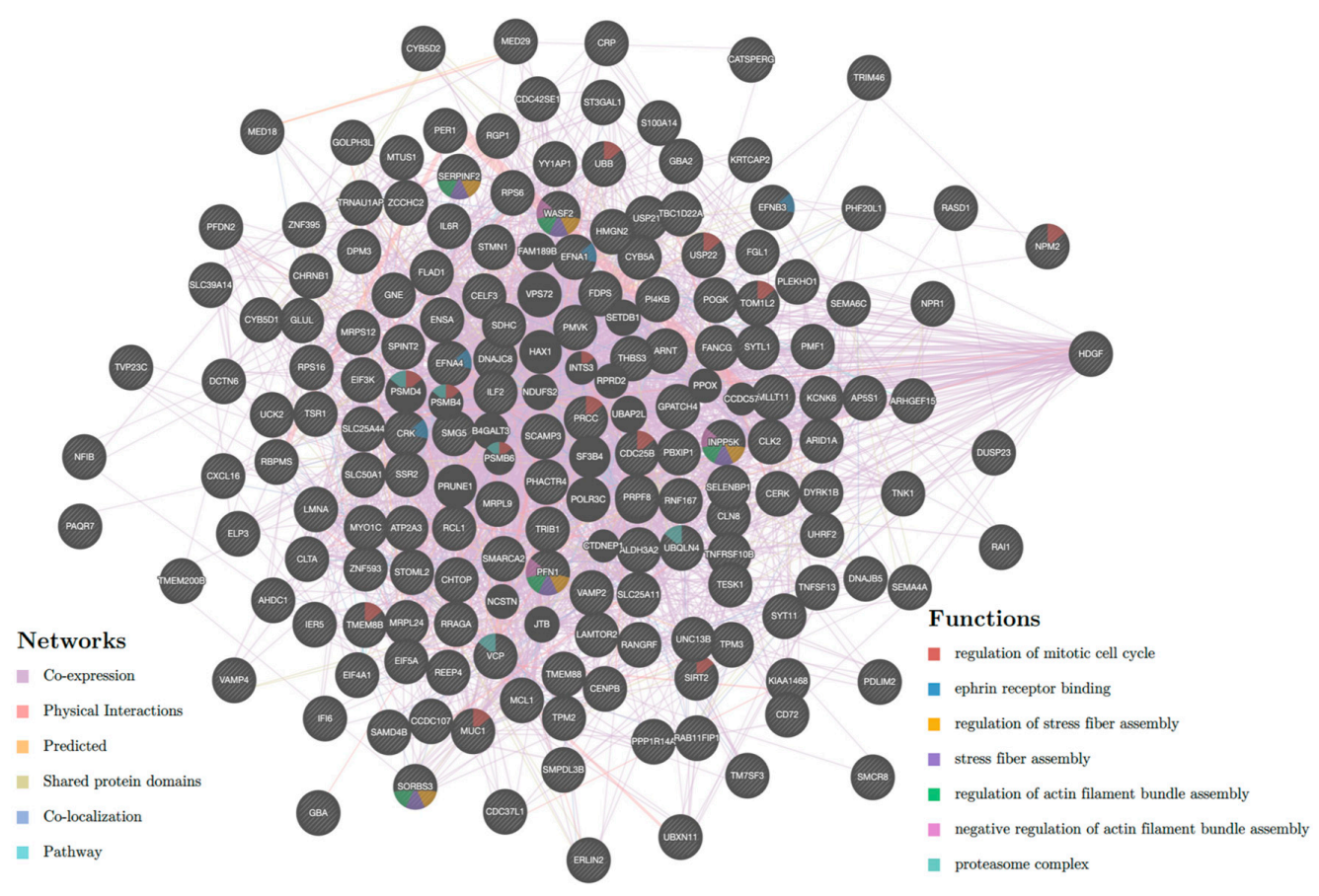

Figure 3. Pathway enrichment analysis performed using software Enrichr on 169 genes regulated by enhancers. 
a.

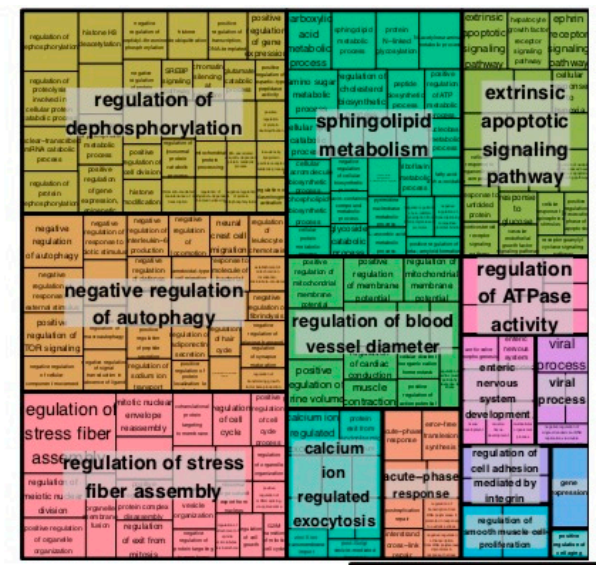

b.

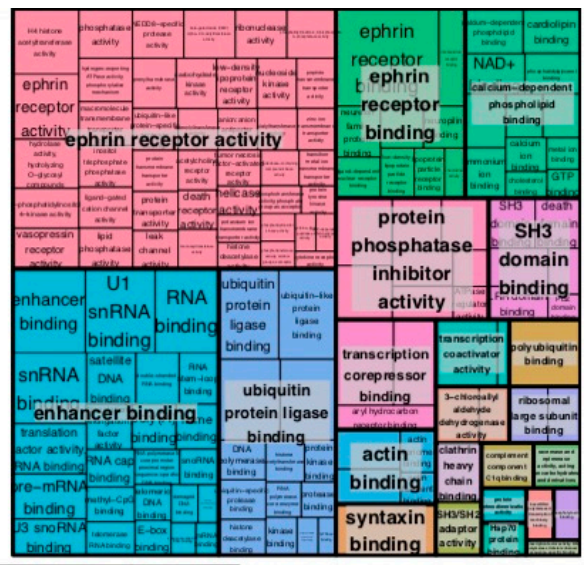

c.

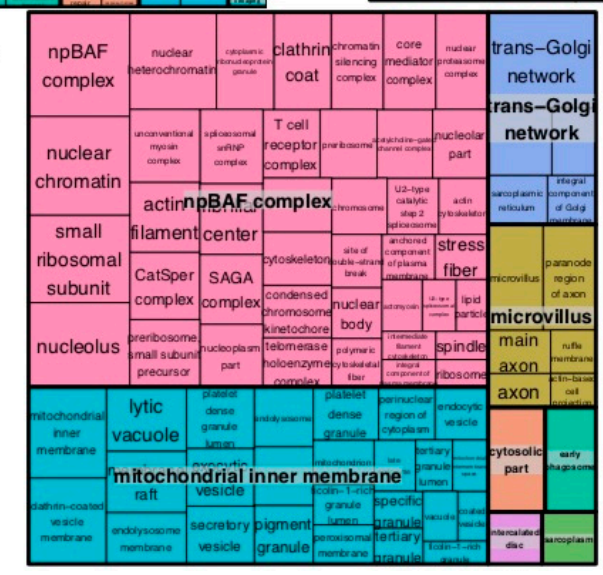

Figure 4. Tree map gene ontology analysis of enhancer CNV associated genes: (a) biological process; (b) molecular function; and, (c) cellular component.

Table 1. Cox proportion hazard ratios with $p$-values of the top 15 genes of enhancer regulated genes; genes sorted based on $p$-value.

\begin{tabular}{ccc}
\hline Gene Symbol & Hazard Ratio (Median Value) & $p$-Value \\
\hline VAMP2 & 2.917023 & 0.000221728 \\
\hline GBA2 & 2.641652475 & 0.000497427 \\
\hline RANGRF & 2.512853346 & 0.000794802 \\
\hline CERK & 2.483219821 & 0.001137485 \\
\hline SORBS3 & 2.450014903 & 0.001099829 \\
\hline AP5S1 & 2.375174975 & 0.001570686 \\
\hline INPP5K & 2.317435264 & 0.00206786 \\
\hline SLC25A44 & 2.291295263 & 0.002326752 \\
\hline RNF167 & 2.239662092 & 0.003430129 \\
\hline DPM3 & 2.196709622 & 0.004076415 \\
\hline SEMA6C & 2.192865982 & 0.005221015 \\
\hline NPR1 & 2.170189229 & 0.00497616 \\
\hline SLC25A11 & 2.104895785 & 0.006106619 \\
\hline USP22 & 2.093982578 & 0.006492927 \\
\hline DNAJB5 & 2.058641673 & 0.008559552 \\
\hline
\end{tabular}




\subsection{Prognostic Potential of Regulatory Genes}

The prognostic potential of genes regulated by enhancer $\mathrm{CNV}$ was identified using data from the human prognostic database PRECOG [32]. Genes with a Z-score $>1.96$ can be evaluated as a prognostic biomarker as this is equivalent to a two tailed $p$-value $<0.05$. We identified 15 genes of the 169 concordant regulatory pair genes having a z-score greater than 1.96, as shown in Table 2 and Table S4. Among the prognostic genes, several genes are involved in a number of pathways regulating pancreatic cancer development: the gene UCK2 is vital for regulating apoptosis; the HDGF gene is considered a jack of all trades in cancer [33]; SSR2 gene inhibition lowers cancer loads in vitro [34]; the USP21 gene is the master regulator of the Hippo pathway [35]; MUC16 regulates pancreatic cancer cell metastasis [36]; and, the STOML2 gene is a prognostic biomarker for pancreatic cancer [37].

Table 2. Enhancer regulated genes present in the PRECOG database with z-score greater than 1.96. Z-scores of these genes were taken from the PRECOG database.

\begin{tabular}{ccc}
\hline Gene & Name & Z-Score \\
\hline CLK2 & CDC-like kinase 2 & 2.39128 \\
\hline EIF4A1 & Eukaryotic translation initiation factor 4A1 & 2.24365 \\
\hline FDPS & Farnesyl diphosphate synthase & 4.03062 \\
\hline FLAD1 & FAD1 flavin adenine dinucleotide synthetase homolog & 2.95895 \\
\hline HDGF & Hepatoma-derived growth factor & 2.16932 \\
\hline ILF2 & Interleukin enhancer binding factor 2, 45kDa & 2.80112 \\
\hline MLLT11 & Myeloid/lymphoid or mixed-lineage leukemia (trithorax homolog & 2.43407 \\
\hline PFN1 & Drosophila); translocated to, 11 & 2.49119 \\
\hline PRCC & Profilin 1 & 3.93837 \\
\hline REEP4 & Papillary renal cell carcinoma (translocation-associated) & 2.98392 \\
\hline$R U S C 1$ & Receptor accessory protein 4 & 3.28846 \\
\hline$S C A M P 3$ & RUN and SH3 domain containing 1 & 2.32083 \\
\hline$U$ UBB & Secretory carrier membrane protein 3 & 2.54009 \\
\hline$U B Q L N 4$ & In multiple clusters & 2.79194 \\
\hline USP21 & Ubiquilin 4 & 2.40871 \\
\hline
\end{tabular}

\subsection{Genes Regulated by Multiple Enhancers or Super Enhancers}

It has been observed that multiple enhancers or clusters of enhancers regulate a single gene. A gene regulated by multiple enhancers is called a gene regulated by super enhancers. We identified 16 genes which are regulated by super enhancers; the Ensembl ID of genes and genomic coordinates of its enhancers are shown in Table 3. There are two main types of aberrant super-enhancers found in various cancers: those involving mutations generated in super-enhancers and those involving the acquisition of new oncogenic super-enhancers. In our study we have identified super-enhancer regions that are created due to the amplification event. These are the master regulators of the cell's fate and identity. Inhibition of super-enhancers seems to be an effective therapy for lowering the burden of cancer, and overexpression of genes regulated by these can act as a diagnostic marker for cancer progression [38]. 
Table 3. Genes regulated by super enhancers, including name of the gene, the genome coordinates of its enhancers and name of the chromosome.

\begin{tabular}{|c|c|c|}
\hline Gene Ensembl ID & Enhancer Coordinates & Chromosome \\
\hline ENSG00000010278 & 6337690-6341770;6387100-6388270;6449480-6450940;6579320-6579700 & Chromosome 12 \\
\hline ENSG00000068745 & 49028850-49029210;49044960-49046260;49044960-49046260;49486410-49486940;49486410-49486940 & Chromosome 3 \\
\hline ENSG00000099622 & 1248660-1249890;1248660-1249890;1248660-1249890;1250530-1251560;1259140-1263220;1275830-1277180;1275830-1277180;1275830-1277180;1275830-1277180 & Chromosome 19 \\
\hline ENSG00000111319 & 6444030-6449420;6449480-6450940;6449480-6450940;6662010-6662810 & Chromosome 12 \\
\hline ENSG00000111674 & 6662010-6662810;6999750-7001150;6999750-7001150;7046720-7047010;7047230-7047600 & Chromosome 12 \\
\hline ENSG00000114353 & 50126460-50126820;50328720-50329650;50358380-50358800;50359200-50359520;50359200-50359520 & Chromosome 3 \\
\hline ENSG00000116285 & 8181000-8181800;8181000-8181800;8193790-8194520;8318930-8320010 & Chromosome 1 \\
\hline ENSG00000116473 & 112134680-112136260;112134680-112136260;112134680-112136260;112202750-112203810 & Chromosome 1 \\
\hline ENSG00000117632 & 26221860-26223380;26221860-26223380;26323300-26324510;26452760-26454880 & Chromosome 1 \\
\hline ENSG00000129968 & 1875340-1876950;1905440-1906150;1905440-1906150;2042030-2042430;2166660-2167720;2579200-2579650 & Chromosome 19 \\
\hline ENSG00000130005 & 1040200-1040500;1040580-1040860;1383940-1385280;1407800-1410200 & Chromosome 19 \\
\hline ENSG00000137154 & 19183280-19185190;19232860-19233120;19379620-19380190;19456550-19457860 & Chromosome 9 \\
\hline ENSG00000142910 & 32013850-32015900;32109070-32109980;32109070-32109980;111308340-111308980;111948770-111949470 & Chromosome 1 \\
\hline ENSG00000143294 & 156659170-156659710;156716040-156721140;156659170-156659710;156716040-156721140 & Chromosome 1 \\
\hline
\end{tabular}




\subsection{Enhancer Expression Correlation with Patient Survival}

To unravel the complex relationship between expression and survival, we analyzed the enhancer expression correlation with patient survival, to display the positive or negative correlation between the two. The enhancer locations, which show the maximum divergence between cancer and the normal sample, are listed in Table 4 and Table S5.

Table 4. Correlation between CNV of enhancers and survival of patients in the case of cancer patients and healthy individuals. The red-to-green color gradient shows high to low correlation.

\begin{tabular}{|c|c|c|}
\hline \multirow{2}{*}{ Coordinates of Enhancer on Genome } & \multicolumn{2}{|c|}{ Correlation (CNV vs. Survival Time) } \\
\hline & Cancer & Healthy \\
\hline chr19:2059605-2060167 & 0.49837818 & 0.2314589 \\
\hline chr5:172380663-172381064 & 0.46715342 & 0.16699777 \\
\hline chr9:136999790-136999893 & 0.46557032 & -0.0626969 \\
\hline chr16:85496879-85497321 & 0.45373278 & -0.6866357 \\
\hline chr14:102415545-102415736 & 0.44115981 & 0.2792461 \\
\hline chr5:10352620-10352938 & 0.43075964 & 0.52245209 \\
\hline chr1:234746093-234747674 & 0.42760743 & 0.98845399 \\
\hline chr19:863983-864016 & 0.42492329 & -0.3898893 \\
\hline chr10:31892273-31892723 & 0.42465511 & -0.5260193 \\
\hline chr4:124621616-124621886 & 0.41837579 & 0.17175469 \\
\hline chr5:964244-964536 & -0.1737289 & 0.52446177 \\
\hline chr6:169573934-169574190 & -0.1747893 & -0.7388221 \\
\hline chr22:50980817-50981280 & -0.1776918 & 0.05853257 \\
\hline chr8:128306934-128307283 & -0.1778833 & -0.3632054 \\
\hline chr14:105500629-105500990 & -0.1798565 & 0.11625874 \\
\hline chr15:99992993-99993428 & -0.1823302 & 0.31824116 \\
\hline chrX:100792680-100793554 & -0.1832455 & -0.6606904 \\
\hline chr22:50979060-50979802 & -0.1916855 & 0.01347895 \\
\hline chr18:12306995-12307375 & -0.1946683 & 0.65842313 \\
\hline chr6:169569548-169569688 & -0.1974295 & 0.1498992 \\
\hline chr8:70042378-70042779 & -0.2421095 & 0.22802202 \\
\hline
\end{tabular}

\subsection{Prognostic Potential of Negatively Correlated Genes}

In this study, our major emphasis was on 169 positively correlated genes (data shown in Table 1), thus we used these genes for all analysis, including identification of prognostic biomarkers. The aim was to understand the impact of negatively correlated genes, meaning enhancer copy number loss/gene upregulation or enhancer copy number gain/gene downregulation $\left(\mathrm{E}^{+} \mathrm{G}^{-} / \mathrm{E}^{-} \mathrm{G}^{+}\right)$. These genes are negatively correlated with CNV of the enhancer. In order to understand the prognostic potential of these genes, we computed the Cox proportion hazard ratio with the $p$-value. The performance of the top genes is shown in Table 5 and performance of the remaining genes is shown in Table S6. 
Table 5. Shows Cox proportion hazard ratio with $p$-value of top negatively correlated genes having potential of prognostic biomarkers, genes sorted based on $p$-value.

\begin{tabular}{ccc}
\hline \multicolumn{3}{c}{ Negatively correlated genes $\left(\mathrm{E}^{+} \mathrm{G}^{-} / \mathrm{E}^{-} \mathrm{G}^{+}\right)$} \\
\hline Gene Symbol & Hazard Ratio & $\boldsymbol{p}$-Value \\
\hline MUM1 & 3.558401305 & $1.33 \times 10^{-5}$ \\
\hline MUM1.1 & 3.558401305 & $1.33 \times 10^{-5}$ \\
\hline MUM1.2 & 3.558401305 & $1.33 \times 10^{-5}$ \\
\hline RBM6 & 3.444153767 & $1.83 \times 10^{-5}$ \\
\hline MBD3 & 3.027911665 & 0.00010983 \\
\hline TLE2 & 2.983689472 & 0.000127766 \\
\hline TLE2.1 & 2.983689472 & 0.000127766 \\
\hline PLD3 & 2.853819471 & 0.00022269 \\
\hline PLD3.1 & 2.853819471 & 0.00022269 \\
\hline$L R P 3$ & 2.81420074 & 0.00030683 \\
\hline
\end{tabular}

\section{Conclusions and Discussion}

Gene expression and cell phenotype are governed by a complex set of regulatory circuits and enhancer elements, which are at the center of governing the fate of each cell cycle. For the better understanding of the disease phenotype and to provide better therapeutics, detailed knowledge of the mechanism of regulation is necessary for every gene whose misexpression is known to cause disease or is involved in disease progression. Due to the non-coding nature of the enhancers, their effect could only be seen through co-expressed genes. Our data integration approach of human enhancers and target provides the first comprehensive DNA-protein interaction in pancreatic cancer. By overlapping the information with $\mathrm{CNV}$, we revealed several important aspects of enhancer deregulation in cancer development. The integration of data from matched TCGA allowed us to propose that the potential mechanism concerning the changes in gene expression is mediated by enhancer copy number gain or loss. We also identified genes (HDGF, UBB) which are upregulated, and downregulation of such genes lowers the cancer load. One such gene is $U B B$, and previous research shows downregulation of the $U B B$ gene via siRNA and its anti-tumor effect in various cell lines and a mouse xenograft model. Thus we recommend downregulation of the $U B B$ gene with enhancer mutation may deliver an anti-tumor effect for pancreatic cancer [26]. A number of super-enhancer linked target genes have been identified. The amplification of enhancers may be used for biomarkers in cancer and may be a potential target for anti-cancer drug design. One of the most fundamental approaches for treating genetic or epigenetic diseases is to disrupt or correct aberrant genomic sequences responsible for the generation of disease-associated enhancers. We also analyze the two gene sets: one shows a positive correlation with enhancers and the other shows a negative correlation with enhancers. From this comparative analysis, we concluded that although enhancers can bring about gene expression change, the survival of a patient with cancer is a multifaceted phenomenon, as the hazard ratio in genes that corresponds to enhancer nature is good but it is also good in genes which do not correspond to enhancer nature. It may be in the particular case of pancreatic cancer that genomic aberration may occur more in the genomic region than the regulatory region. Further study is required to confirm the results of survival of patients and enhancer expression. With recent advances in genome engineering technologies such as TALEN (Transcription activator-like effector nuclease) and CRISPR/Cas9, it is now more convenient to generate mutations in cells or animal models, providing unprecedented opportunities to develop effective gene therapies for enhancer-associated diseases. Moreover, enhancers and super-enhancers can be used as prognostic markers for the prediction of disease risk and progression. Thus, integrative analysis of a gene transcription signature and the enhancer profile of patients or healthy individuals 
could emerge as an important approach for disease diagnosis. We conclude that coupling of enhancer profiles with gene expression changes has possibly unearthed a powerful approach to treat disease, and can be expected to strengthen personalized medicine in the near future.

\section{Materials and Methods}

\subsection{Enhancers and Target Gene for Human Pancreatic Tissue}

In the present study, pancreatic tissue specific enhancers were mined from EnhancerAtlas (http://www.enhanceratlas.org/data/AllEPs/Pancreas_EP.txt). EnhancerAtlas maintains enhancers and associated/target genes of 105 human cell/tissue types. Enhancer sequences with their genomic coordinates mapped to human genome assembly GRCh37/hg19 were downloaded in plain text format. This contained 3876 sequences with genomic coordinates specific to pancreatic tissue. We also downloaded the target genes corresponding to these enhancers from EnhancerAtlas. Enhancers that do not interact with any promoter and gene sequence and with confidence scores below 0.7 were removed. This step resulted in only 1696 enhancers, which were strongly associated with their target gene and thus regulation of gene expression. These 1696 strong enhancers form 2968 interaction pairs. Figure 5 shows the overall mind map and computational workflow used to carry out this study.

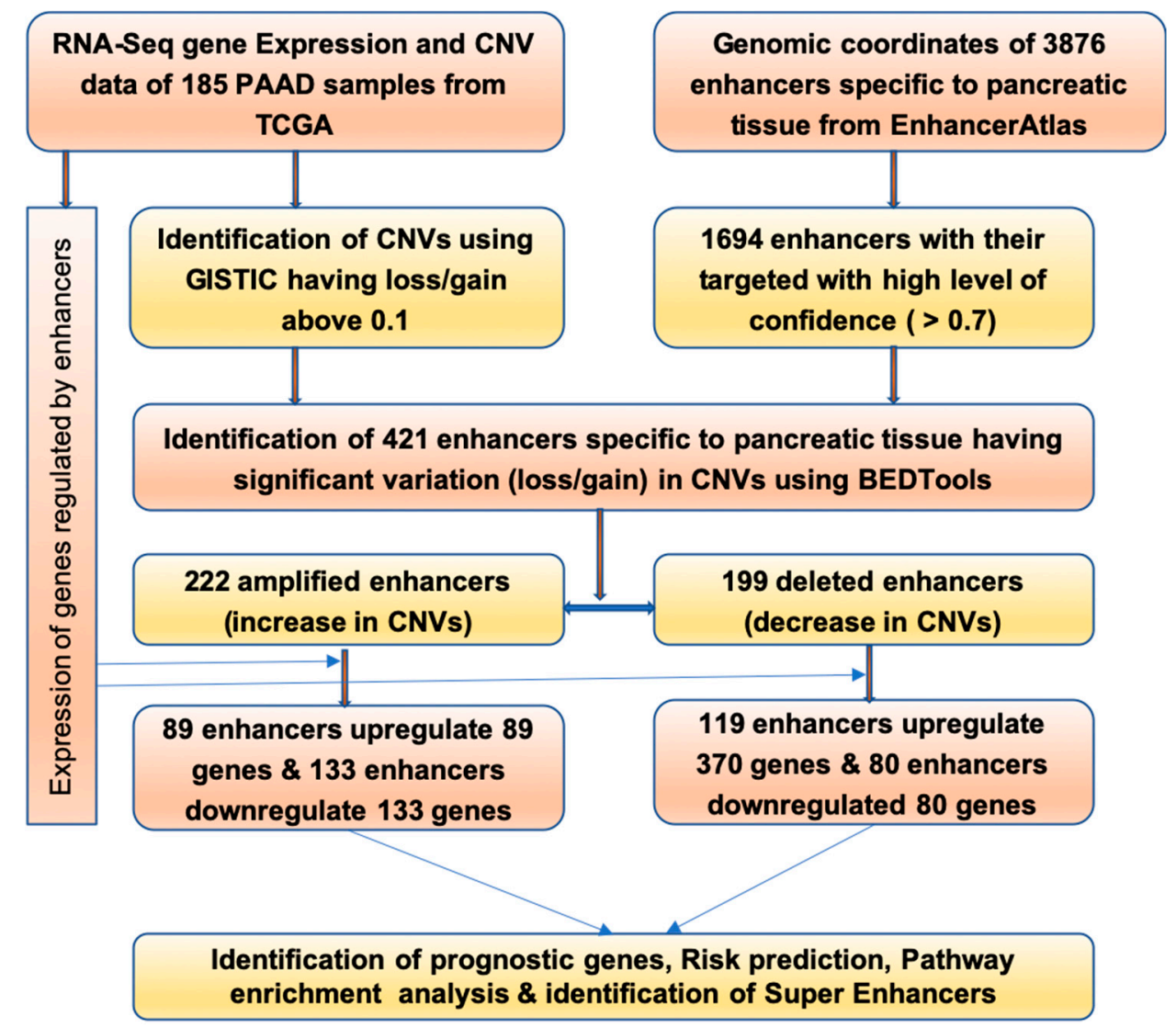

Figure 5. Computational workflow of the study.

\subsection{Pancreatic Cancer Dataset from TCGA}

The pancreatic adenocarcinoma (PAAD) dataset of copy number variations and gene expression levels of 185 samples was downloaded from The Cancer Genome Atlas (TCGA) database (https: //portal.gdc.cancer.gov/projects/TCGA-PAAD). Out of 185 samples, 181 correspond to tumor samples and four samples consist of normal samples derived from adjacent solid tissue. More precisely, Illumina HiSeq RNASeqV2 in 728 files and copy number variations in 737 files were taken into consideration. 


\subsection{Copy Number Variations Identification}

We implemented the GISTIC2.0 [39] (Genomic Identification of Significant Targets in Cancer, version 2.0) algorithm to identify the regions of the genome that were amplified or deleted across the samples. This algorithm uses human genome assembly $\mathrm{Hg} 19$ as the reference genome, which consists of information on recurrent CNV cytoband and gene location. Thresholds were set as default GISTIC2.0 parameters. The threshold for copy number gain/loss was set at 0.1 , so that regions with a copy number value above 0.1 were acknowledged as a copy number gain (i.e., amplification), and regions with a copy number value below 0.1 were acknowledged as a copy number loss (i.e., deletion) [40]. Segments that comprised less than four markers were combined with the neighboring segment nearest in copy number; regions with q-values less than 0.25 were acknowledged as significant. We then used genomic intersecting functionality from BEDTools [41] to intersect each recurrent significant CNV identified from GISTIC to map onto the genomic coordinates of enhancers of pancreatic tissue. This step led to the identification of recurrent significant $\mathrm{CNV}$ of enhancers of pancreatic tissue.

\subsection{Screening for Differential Gene Expression}

Differential gene expression (DGE) analysis on the mRNA transcripts was done after quantile filtration, which was achieved using the quantile method and a cut-off value of 0.25 . To ascertain whether a gene was expressed differentially, the test of the hypothesis, with the fold-change between the two conditions as normal and tumor conditions, was calculated. We applied the TCGAanalyze_DEA function, which performs DEA using various functions of the edgeR package from Bioconductor [42]. The function edgeR:exactTest makes pair-wise tests for differential expression between two groups. The $p$-values obtained from the exactTest sorted in ascending order were corrected/adjusted using the false discovery rate (FDR) correction which returns the top differentially expressed genes. Thresholds for $\log$ fold change $(\log F C)$ and FDR were set at 1 and 0.1 , respectively, such that differentially expressed mRNAs were considered to be significant if $\log F C>1$ and FDR $<0.05$.

\subsection{Combination of Gene Expression and Copy Number Variation}

The identification of differentially expressed genes with CNVs such as gain or loss was accomplished here, by considering the outputs of the gene expression analysis, so that, if the gene was upregulated or downregulated, and of CNV analysis, that is, if the region was amplified or deleted. We selected the upregulated genes with an amplified copy number and downregulated genes with copy number deletion in their corresponding regulatory enhancer regions of pancreatic adenocarcinoma patients. These genes correspond with the nature of the enhancer element.

\subsection{Gene Network Construction, GO Enrichment and Super Enhancer Based Regulation}

In order to understand the significance of both upregulated and downregulated genes, the STRING database [43] was used to construct the gene network of enhancer CNV driven genes at FDR 0.25. GO enrichment analysis of genes was done with the help of Enrichr, an R package [44]. We also aimed to identify super-enhancer-based gene regulation. Super-enhancers were defined as multiple enhancers regulating the same gene expression with an upregulating effect [45].

\subsection{Correlation Analysis of Enhancer Expression with Patient Survival}

Enhancer elements specifically expressed in pancreatic tissue were taken from study [3]. Pancreatic adenocarcinoma clinical files were downloaded from TCGA and correlation for enhancer expression and patient survival was done using in-house Python scripts.

Supplementary Materials: Supplementary materials can be found at http://www.mdpi.com/1422-0067/20/14/ 3582/s1.

Author Contributions: G.P.S.R. made substantial contributions to the conception and design of the work, to data analysis, supervision, funding acquisition, project administration and in drafting the manuscript. R.K., 
S.P. and V.K. made substantial contributions to the data curation, formal analysis and interpretation of the data. R.K., S.P. and G.N. made substantial contribution in Methodology, original draft preparation, review and editing and visualization.

Funding: This work was supported by J. C. Bose Fellowship (Grant number SRP076), Department of Science and Technology, India.

Acknowledgments: Authors are thankful to funding agencies Department of Biotechnology (DBT) and University Grant Commission (UGC) and Council of Scientific and Industrial Research (CSIR), Govt. of India for financial support and fellowships. We are also thankful to Anjali Lathwal, our lab colleague for providing in time support with enthusiasm whenever required.

Conflicts of Interest: The authors declare no conflict of interest.

\section{Abbreviations}

TCGA The Cancer Genome Atlas

ICGC International Cancer Genome Consortium

DGE Differential Gene Expression

CNV Copy Number variation

PAAD Pancreatic Adenocarcinoma

CAF cancer-associated fibroblasts

COSMIC Catalogue of Somatic Mutation in Cancer

GISTIC2.0 Genomic Identification of Significant Target in Cancer

\section{References}

1. Wong, M.C.S.; Jiang, J.Y.; Liang, M.; Fang, Y.; Yeung, M.S.; Sung, J.J.Y. Global temporal patterns of pancreatic cancer and association with socioeconomic development. Sci. Rep. 2017, 7, 3165. [CrossRef] [PubMed]

2. Chang, J.S.; Chen, L.-T.; Shan, Y.-S.; Chu, P.-Y.; Tsai, C.-R.; Tsai, H.-J. The incidence and survival of pancreatic cancer by histology, including rare subtypes: A nation-wide cancer registry-based study from Taiwan. Cancer Med. 2018, 7, 5775-5788. [CrossRef] [PubMed]

3. Chen, H.; Li, C.; Peng, X.; Zhou, Z.; Weinstein, J.N.; Cancer Genome Atlas Research Network, H.; Liang, H.; Demchok, J.A.; Felau, I.; Kasapi, M.; et al. A Pan-Cancer Analysis of Enhancer Expression in Nearly 9000 Patient Samples. Cell 2018, 173, 386-399.e12. [CrossRef] [PubMed]

4. Grossman, R.L.; Heath, A.P.; Ferretti, V.; Varmus, H.E.; Lowy, D.R.; Kibbe, W.A.; Staudt, L.M. Toward a Shared Vision for Cancer Genomic Data. N. Engl. J. Med. 2016, 375, 1109-1112. [CrossRef] [PubMed]

5. International Cancer Genome Consortium; Hudson, T.J.; Anderson, W.; Artez, A.; Barker, A.D.; Bell, C.; Bernabé, R.R.; Bhan, M.K.; Calvo, F.; Eerola, I.; et al. International network of cancer genome projects. Nature 2010, 464, 993-998. [PubMed]

6. Forbes, S.A.; Beare, D.; Boutselakis, H.; Bamford, S.; Bindal, N.; Tate, J.; Cole, C.G.; Ward, S.; Dawson, E.; Ponting, L.; et al. COSMIC: Somatic cancer genetics at high-resolution. Nucleic Acids Res. 2017, 45, D777-D783. [CrossRef] [PubMed]

7. Araya, C.L.; Cenik, C.; Reuter, J.A.; Kiss, G.; Pande, V.S.; Snyder, M.P.; Greenleaf, W.J. Identification of significantly mutated regions across cancer types highlights a rich landscape of functional molecular alterations. Nat. Genet. 2016, 48, 117-125. [CrossRef]

8. Maurano, M.T.; Humbert, R.; Rynes, E.; Thurman, R.E.; Haugen, E.; Wang, H.; Reynolds, A.P.; Sandstrom, R.; $\mathrm{Qu}, \mathrm{H}$.; Brody, J.; et al. Systematic localization of common disease-associated variation in regulatory DNA. Science 2012, 337, 1190-1195. [CrossRef]

9. Diederichs, S.; Bartsch, L.; Berkmann, J.C.; Fröse, K.; Heitmann, J.; Hoppe, C.; Iggena, D.; Jazmati, D.; Karschnia, P.; Linsenmeier, M.; et al. The dark matter of the cancer genome: Aberrations in regulatory elements, untranslated regions, splice sites, non-coding RNA and synonymous mutations. EMBO Mol. Med. 2016, 8, 442-457. [CrossRef]

10. Perera, D.; Chacon, D.; Thoms, J.A.I.; Poulos, R.C.; Shlien, A.; Beck, D.; Campbell, P.J.; Pimanda, J.E.; Wong, J.W.H. OncoCis: Annotation of cis-regulatory mutations in cancer. Genome Biol. 2014, 15, 485. 
11. Hornshøj, H.; Nielsen, M.M.; Sinnott-Armstrong, N.A.; Świtnicki, M.P.; Juul, M.; Madsen, T.; Sallari, R.; Kellis, M.; Ørntoft, T.; Hobolth, A.; et al. Pan-cancer screen for mutations in non-coding elements with conservation and cancer specificity reveals correlations with expression and survival. NPJ Genomic Med. 2018, 3, 1. [CrossRef] [PubMed]

12. Herz, H.-M. Enhancer deregulation in cancer and other diseases. BioEssays 2016, 38, 1003-1015. [CrossRef] [PubMed]

13. Herman-Izycka, J.; Wlasnowolski, M.; Wilczynski, B. Taking promoters out of enhancers in sequence based predictions of tissue-specific mammalian enhancers. BMC Med. Genomics 2017, 10, 34. [CrossRef] [PubMed]

14. Symmons, O.; Spitz, F. From remote enhancers to gene regulation: Charting the genome's regulatory landscapes. Philos. Trans. R. Soc. Lond. B. Biol. Sci. 2013, 368, 20120358. [CrossRef] [PubMed]

15. Zhang, G.; Shi, J.; Zhu, S.; Lan, Y.; Xu, L.; Yuan, H.; Liao, G.; Liu, X.; Zhang, Y.; Xiao, Y.; et al. DiseaseEnhancer: A resource of human disease-associated enhancer catalog. Nucleic Acids Res. 2018, 46, D78-D84. [CrossRef] [PubMed]

16. Gamazon, E.R.; Stranger, B.E. The impact of human copy number variation on gene expression. Brief. Funct. Genomics 2015, 14, 352-357. [CrossRef]

17. Jia, Y.; Chen, L.; Jia, Q.; Dou, X.; Xu, N.; Liao, D.J. The well-accepted notion that gene amplification contributes to increased expression still remains, after all these years, a reasonable but unproven assumption. J. Carcinog. 2016, 15, 3. [CrossRef] [PubMed]

18. Zhang, X.; Choi, P.S.; Francis, J.M.; Imielinski, M.; Watanabe, H.; Cherniack, A.D.; Meyerson, M. Identification of focally amplified lineage-specific super-enhancers in human epithelial cancers. Nat. Genet. 2016, 48, 176-182. [CrossRef]

19. Zhang, X.; Choi, P.S.; Francis, J.M.; Gao, G.F.; Campbell, J.D.; Ramachandran, A.; Mitsuishi, Y.; Ha, G.; Shih, J.; Vazquez, F.; et al. Somatic Superenhancer Duplications and Hotspot Mutations Lead to Oncogenic Activation of the KLF5 Transcription Factor. Cancer Discov. 2018, 8, 108-125. [CrossRef]

20. Gao, T.; He, B.; Liu, S.; Zhu, H.; Tan, K.; Qian, J. EnhancerAtlas: A resource for enhancer annotation and analysis in 105 human cell/tissue types. Bioinformatics 2016, 32, 3543-3551. [CrossRef]

21. He, X.; Fuller, C.K.; Song, Y.; Meng, Q.; Zhang, B.; Yang, X.; Li, H. Sherlock: Detecting Gene-Disease Associations by Matching Patterns of Expression QTL and GWAS. Am. J. Hum. Genet. 2013, 92, 667-680. [CrossRef] [PubMed]

22. Hu, B.; Shi, C.; Jiang, H.-X.; Qin, S.-Y. Identification of novel therapeutic target genes and pathway in pancreatic cancer by integrative analysis. Medicine (Baltimore). 2017, 96, e8261. [CrossRef] [PubMed]

23. Basso, D. Altered glucose metabolism and proteolysis in pancreatic cancer cell conditioned myoblasts: Searching for a gene expression pattern with a microarray analysis of 5000 skeletal muscle genes. Gut 2004, 53, 1159-1166. [CrossRef] [PubMed]

24. Zhang, X.; Sheng, J.; Zhang, Y.; Tian, Y.; Zhu, J.; Luo, N.; Xiao, C.; Li, R. Overexpression of SCAMP3 is an indicator of poor prognosis in hepatocellular carcinoma. Oncotarget 2017, 8, 109247-109257. [CrossRef] [PubMed]

25. Sahraei, M.; Roy, L.D.; Curry, J.M.; Teresa, T.L.; Nath, S.; Besmer, D.; Kidiyoor, A.; Dalia, R.; Gendler, S.J.; Mukherjee, P. MUC1 regulates PDGFA expression during pancreatic cancer progression. Oncogene 2012, 31, 4935-4945. [CrossRef] [PubMed]

26. Oh, C.; Park, S.; Lee, E.K.; Yoo, Y.J. Downregulation of ubiquitin level via knockdown of polyubiquitin gene Ubb as potential cancer therapeutic intervention. Sci. Rep. 2013, 3, 2623. [CrossRef] [PubMed]

27. Xia, P.; Xu, X.-Y. PI3K/Akt/mTOR signaling pathway in cancer stem cells: From basic research to clinical application. Am. J. Cancer Res. 2015, 5, 1602-1609. [PubMed]

28. Laplante, M.; Sabatini, D.M. mTOR signaling at a glance. J. Cell Sci. 2009, 122, 3589-3594. [CrossRef] [PubMed]

29. Bhullar, K.S.; Lagarón, N.O.; McGowan, E.M.; Parmar, I.; Jha, A.; Hubbard, B.P.; Rupasinghe, H.P.V. Kinase-targeted cancer therapies: Progress, challenges and future directions. Mol. Cancer 2018, 17, 48. [CrossRef]

30. Messenger, S.W.; Jones, E.K.; Holthaus, C.L.; Thomas, D.D.H.; Cooley, M.M.; Byrne, J.A.; Mareninova, O.A.; Gukovskaya, A.S.; Groblewski, G.E. Acute acinar pancreatitis blocks vesicle-associated membrane protein 8 (VAMP8)-dependent secretion, resulting in intracellular trypsin accumulation. J. Biol. Chem. 2017, 292, 7828-7839. [CrossRef] 
31. Rivera, I.-G.; Ordoñez, M.; Presa, N.; Gangoiti, P.; Gomez-Larrauri, A.; Trueba, M.; Fox, T.; Kester, M.; Gomez-Muñoz, A. Ceramide 1-phosphate regulates cell migration and invasion of human pancreatic cancer cells. Biochem. Pharmacol. 2016, 102, 107-119. [CrossRef] [PubMed]

32. Gentles, A.J.; Newman, A.M.; Liu, C.L.; Bratman, S.V.; Feng, W.; Kim, D.; Nair, V.S.; Xu, Y.; Khuong, A.; Hoang, C.D.; et al. The prognostic landscape of genes and infiltrating immune cells across human cancers. Nat. Med. 2015, 21, 938-945. [CrossRef] [PubMed]

33. Bao, C.; Wang, J.; Ma, W.; Wang, X.; Cheng, Y. HDGF: A novel jack-of-all-trades in cancer. Future Oncol. 2014, 10, 2675-2685. [CrossRef] [PubMed]

34. Fisher, W.E.; Wu, Y.; Amaya, F.; Berger, D.H. Somatostatin receptor subtype 2 gene therapy inhibits pancreatic cancer in vitro. J. Surg. Res. 2002, 105, 58-64. [CrossRef] [PubMed]

35. Nguyen, H.T.; Kugler, J.-M.; Loya, A.C.; Cohen, S.M. USP21 regulates Hippo pathway activity by mediating MARK protein turnover. Oncotarget 2017, 8, 64095-64105. [CrossRef] [PubMed]

36. Muniyan, S.; Haridas, D.; Chugh, S.; Rachagani, S.; Lakshmanan, I.; Gupta, S.; Seshacharyulu, P.; Smith, L.M.; Ponnusamy, M.P.; Batra, S.K. MUC16 contributes to the metastasis of pancreatic ductal adenocarcinoma through focal adhesion mediated signaling mechanism. Genes Cancer 2016, 7, 110-124. [PubMed]

37. Takadate, T.; Onogawa, T.; Fukuda, T.; Motoi, F.; Suzuki, T.; Fujii, K.; Kihara, M.; Mikami, S.; Bando, Y.; Maeda, S.; et al. Novel prognostic protein markers of resectable pancreatic cancer identified by coupled shotgun and targeted proteomics using formalin-fixed paraffin-embedded tissues. Int. J. Cancer 2013, 132, 1368-1382. [CrossRef]

38. Shin, H.Y. Targeting Super-Enhancers for Disease Treatment and Diagnosis. Mol. Cells 2018, 41, 506-514.

39. Mermel, C.H.; Schumacher, S.E.; Hill, B.; Meyerson, M.L.; Beroukhim, R.; Getz, G. GISTIC2.0 facilitates sensitive and confident localization of the targets of focal somatic copy-number alteration in human cancers. Genome Biol. 2011, 12, R41. [CrossRef]

40. Kaveh, F.; Baumbusch, L.O.; Nebdal, D.; Børresen-Dale, A.-L.; Lingjærde, O.C.; Edvardsen, H.; Kristensen, V.N.; Solvang, H.K. A systematic comparison of copy number alterations in four types of female cancer. BMC Cancer 2016, 16, 913. [CrossRef]

41. Quinlan, A.R.; Hall, I.M. BEDTools: A flexible suite of utilities for comparing genomic features. Bioinformatics 2010, 26, 841-842. [CrossRef] [PubMed]

42. Robinson, M.D.; McCarthy, D.J.; Smyth, G.K. edgeR: A Bioconductor package for differential expression analysis of digital gene expression data. Bioinformatics 2010, 26, 139-140. [CrossRef] [PubMed]

43. Szklarczyk, D.; Morris, J.H.; Cook, H.; Kuhn, M.; Wyder, S.; Simonovic, M.; Santos, A.; Doncheva, N.T.; Roth, A.; Bork, P.; et al. The STRING database in 2017: Quality-controlled protein-protein association networks, made broadly accessible. Nucleic Acids Res. 2017, 45, D362-D368. [CrossRef] [PubMed]

44. Kuleshov, M.V.; Jones, M.R.; Rouillard, A.D.; Fernandez, N.F.; Duan, Q.; Wang, Z.; Koplev, S.; Jenkins, S.L.; Jagodnik, K.M.; Lachmann, A.; et al. Enrichr: A comprehensive gene set enrichment analysis web server 2016 update. Nucleic Acids Res. 2016, 44, W90-W97. [CrossRef] [PubMed]

45. Li, X.; Liu, Y.; Lu, J.; Zhao, M. Integrative analysis to identify oncogenic gene expression changes associated with copy number variations of enhancer in ovarian cancer. Oncotarget 2017, 8, 91558-91567. [CrossRef] [PubMed]

(C) 2019 by the authors. Licensee MDPI, Basel, Switzerland. This article is an open access article distributed under the terms and conditions of the Creative Commons Attribution (CC BY) license (http://creativecommons.org/licenses/by/4.0/). 